R. Chapko et al. / Electronic Journal of Boundary Elements, Vol. 9, No. 1, pp. 1-15 (2011)

\title{
Recovering boundary data in planar heat conduction using a boundary integral equation method
}

\author{
R. Chapko ${ }^{1}$ B. T. Johansson ${ }^{2}$ and V. Vavrychuk ${ }^{1}$ \\ ${ }^{1}$ Faculty of Applied Mathematics and Informatics, Ivan Franko National \\ University of Lviv, 79000 Lviv, Ukraine \\ e-mail: chapko@is.lviv.ua \\ ${ }^{2}$ School of Mathematics, University of Birmingham, Edgbaston, Birmingham \\ B15 2TT, UK \\ e-mail: b.t.johansson@bham.ac.uk
}

\begin{abstract}
We consider a Cauchy problem for the heat equation, where the temperature field is to be reconstructed from the temperature and heat flux given on a part of the boundary of the solution domain. We employ a Landweber type method proposed in [2], where a sequence of mixed well-posed problems are solved at each iteration step to obtain a stable approximation to the original Cauchy problem. We develop an efficient boundary integral equation method for the numerical solution of these mixed problems, based on the method of Rothe. Numerical examples are presented both with exact and noisy data, showing the efficiency and stability of the proposed procedure and approximations.
\end{abstract}

Keywords: Cauchy problem; Heat equation; Rothe's method; Boundary integral equation method; Trigonometrical quadrature.

\section{Introduction}

We assume that we have a two-dimensional conducting body modelled by a doubly connected domain $D$ in $\mathbb{R}^{2}$ with sufficiently smooth boundary, consisting of the internal boundary $\Gamma_{1}$ and the external boundary $\Gamma_{2}$, both being simple closed curves, see further Figure 1.

In practical engineering applications, the external boundary $\Gamma_{2}$ might be accessible for measurements while the internal boundary is inaccessible. Thus, one can obtain overspecified data on $\Gamma_{2}$, while leaving values on $\Gamma_{1}$ unknown. Assuming that the temperature and heat flux have been measured on $\Gamma_{2}$, we are interested in reconstructing the missing temperature values on the internal boundary $\Gamma_{1}$. Supposing that the heat flow is such that it can be modeled by the linear heat 


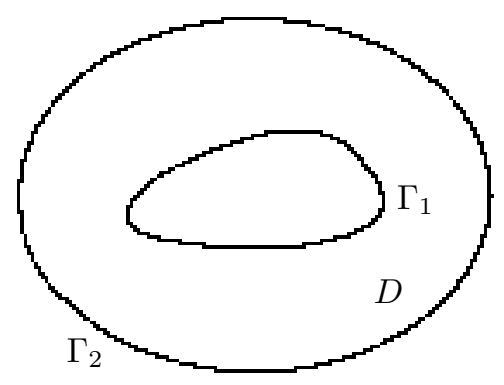

Figure 1: An example of the solution domain $D$ and its boundary parts

equation, the temperature field $u$ is a solution to

$$
\begin{cases}\frac{1}{c} \frac{\partial u}{\partial t}=\Delta u & \text { in } D \times(0, T), \\ u=f_{1} & \text { on } \Gamma_{2} \times(0, T), \\ \frac{\partial u}{\partial \nu}=f_{2} & \text { on } \Gamma_{2} \times(0, T), \\ u(x, 0)=0 & \text { for } x \in D,\end{cases}
$$

where the functions $f_{1}$ and $f_{2}$ are given and sufficiently smooth, $\nu$ is the unit outward normal to the boundary and the constant $c$ is the thermal diffusivity. For simplicity, we have chosen the initial temperature distribution to be zero. Uniqueness of a solution to this Cauchy problem is well-known, see, for example, [15]. We shall assume that data are given such that there exists a solution. However, this solution will not depend continuously on the data, i.e. the problem is ill-posed and regularizing methods are needed for the stable approximation.

In the stationary case, there are numerous papers which solve the corresponding Cauchy problem via iterative methods based on the ideas presented in [10, 11]. However, to the authors knowledge, for the time-dependent case, considerably fewer works based on such iterative methods have been presented [1, 2, 7, 14]. Mainly the finite element method (FEM) has been employed for the numerical implementation. Since only boundary data are needed in these iterative procedures, using FEM based solvers can make the iterations slow since all of the solution domain has to be discretized.

We focus in this work on the numerical implementation of a method introduced in [2] and we shall develop a boundary integral technique where only boundary data are involved, making the iterations faster. That such iterative boundary integral methods can be effective for Cauchy problems was shown in [3] and we now extend these ideas to the time-dependent case.

For the outline of this work, in Section 2 we recall the iterative procedure and some of its properties. In Section 3, we develop an efficient numerical boundary integral technique based on the method of Rothe for solving mixed boundary value problems for the heat equation in annular domains. We then give numerical results 
in Section 4 for various data, both exact and with noise, and for several different solution domains.

\section{The iterative procedure}

We define two operators needed to explain properties of the iterative procedure. First, for every $\eta \in L^{2}\left(\Gamma_{1} \times(0, T)\right)$, the operator $K$ is defined as $K \eta:=\left.u\right|_{\Gamma_{2} \times(0, T)}$ where $u$ is the solution of the following mixed boundary value problem

$$
\begin{cases}\frac{1}{c} \frac{\partial u}{\partial t}-\Delta u=0 & \text { in } D \times(0, T), \\ u=\eta & \text { on } \Gamma_{1} \times(0, T), \\ \frac{\partial u}{\partial \nu}=0 & \text { on } \Gamma_{2} \times(0, T) \\ u(x, 0)=0 & \text { for } x \in D .\end{cases}
$$

Then, for every $f_{2} \in L^{2}\left(\Gamma_{2} \times(0, T)\right)$ the operator $G$ is defined as $G f_{2}:=\left.u\right|_{\Gamma_{2} \times(0, T)}$ where $u$ is the solution of the following mixed problem

$$
\begin{cases}\frac{1}{c} \frac{\partial u}{\partial t}-\Delta u=0 & \text { in } D \times(0, T), \\ u=0 & \text { on } \Gamma_{1} \times(0, T), \\ \frac{\partial u}{\partial \nu}=f_{2} & \text { on } \Gamma_{2} \times(0, T), \\ u(x, 0)=0 & \text { for } x \in D .\end{cases}
$$

Note that if we can find $\eta$ such that $K \eta+G f_{2}=f_{1}$, we have a solution to the Cauchy problem (1.1). Thus, we have to solve the equation

$$
K \eta=f_{1}-G f_{2} .
$$

In [2], the operator $K$ was investigated and it was shown that the kernel consists of the element 0 only and that its inverse is unbounded in $L^{2}$. Thus, to solve (2.3) in a stable way regularizing methods are needed. One possibility is a Landweber type procedure of the following form ([2]):

- First we choose an arbitrary initial approximation $\eta_{0}$ of the (unknown) function $\left.u\right|_{\Gamma_{1} \times(0, T)}$, where $u$ satisfies (1.1).

- The value $\left.u_{0}\right|_{\Gamma_{2} \times(0, T)}$ is calculated from the solution of the Dirichlet-Neumann mixed initial boundary value problem.

$$
\begin{cases}\frac{1}{c} \frac{\partial u_{0}}{\partial t}-\Delta u_{0}=0 & \text { in } D \times(0, T) \\ u_{0}=\eta_{0} & \text { on } \Gamma_{1} \times(0, T) \\ \frac{\partial u_{0}}{\partial \nu}=f_{2} & \text { on } \Gamma_{2} \times(0, T) \\ u_{0}(x, 0)=0 & \text { for } x \in D\end{cases}
$$


- Now the value $\left.\frac{\partial v_{0}}{\partial \nu}\right|_{\Gamma_{1} \times(0, T)}$ is calculated from the solution of the mixed initial boundary value problem

$$
\begin{cases}\frac{1}{c} \frac{\partial v_{0}}{\partial t}+\Delta v_{0}=0 & \text { in } D \times(0, T) \\ v_{0}=0 & \text { on } \Gamma_{1} \times(0, T) \\ \frac{\partial v_{0}}{\partial \nu}=u_{0}-f_{1} & \text { on } \Gamma_{2} \times(0, T) \\ v_{0}(x, T)=0 & \text { for } x \in D\end{cases}
$$

Knowing $u_{k-1}$ and $v_{k-1}$ we proceed as follows.

- We evaluate $\left.u_{k}\right|_{\Gamma_{2} \times(0, T)}$ by solving the Dirichlet-Neumann problem

$$
\begin{cases}\frac{1}{c} \frac{\partial u_{k}}{\partial t}-\Delta u_{k}=0 & \text { in } D \times(0, T), \\ u_{k}=\eta_{k}=\eta_{k-1}+\gamma_{L} \frac{\partial}{\partial \nu} v_{k-1} & \text { on } \Gamma_{1} \times(0, T), \\ \frac{\partial u_{k}}{\partial \nu}=f_{2} & \text { on } \Gamma_{2} \times(0, T), \\ u_{k}(x, 0)=0 & \text { for } x \in D,\end{cases}
$$

for some value of $\gamma_{L}>0$.

- Further, by solving the Dirichlet-Neumann problem

$$
\begin{cases}\frac{1}{c} \frac{\partial v_{k}}{\partial t}+\Delta v_{k}=0 & \text { in } D \times(0, T) \\ v_{k}=0 & \text { on } \Gamma_{1} \times(0, T) \\ \frac{\partial v_{k}}{\partial \nu}=u_{k}-f_{1} & \text { on } \Gamma_{2} \times(0, T) \\ v_{k}(x, T)=0 & \text { for } x \in D\end{cases}
$$

we can find $\left.\frac{\partial v_{k}}{\partial v}\right|_{\Gamma_{1} \times(0, T)}$ and then proceed to the evaluation of the next approximation $u_{k+1}$ by repeating the previous two steps.

The well-posedness of these mixed-problems in an $L^{2}$-setting was given in [2]. Moreover, it was shown there that the procedure can be written as

$$
\eta_{k}=\eta_{k-1}-\gamma_{L} K^{*}\left(K \eta_{k-1}+G f_{2}-f_{1}\right),
$$

where $\gamma_{L}>0$ is the relaxation parameter, which is the classical Landweber method for solving (2.3). Thus, the following holds, see [2] for details:

Theorem 2.1 Let $f_{1}, f_{2} \in L^{2}\left(\Gamma_{2} \times(0, T)\right)$, and let $u$ be the solution to (1.1) and $u_{k}$ be the $k$-th approximation in the above procedure. Provided that $0<\gamma_{L}<$ $1 /\|K\|^{2}$, where $\|\cdot\|$ denotes the standard operator norm, then

$$
\lim _{k \rightarrow \infty}\left\|u-u_{k}\right\|_{L^{2}(D \times(0, T))}=0,
$$

for any initial element $\eta_{0} \in L^{2}\left(\Gamma_{1} \times(0, T)\right)$. 
Note that the discrepancy principle [13] can be employed in the case of noisy data. It is known that the Landweber iteration has slow convergence and the choice of the regularizing parameter does influence the rate of convergence as well. In a future study we aim to implement more advanced iterative schemes such as conjugate gradient type methods to improve the rate of convergence.

Note also that if the boundary of the solution domain has corner points, derivatives of the solution might not belong to the $L^{2}$ space eventhough the given data are smooth. Instead, the analysis has to be performed in weighted spaces of Kondrat'ev [9] and the iterative scheme adjusted to contain weights. This type of a weighted iterative scheme is presented and analysed in [8] and can be employed in the case of corner points of the solution domain.

\section{Numerical solution of the mixed boundary value problems}

For the solution of the direct mixed problems needed in the procedure given in the previous section, we use a combination the method of Rothe for semi-discretization in time and a boundary integral equation method for discretization with respect to the spatial variables.

\subsection{Rothe's method and boundary integral equations}

Let us define the following uniform grid on the time-interval $[0, T)$

$$
t_{n}=(n+1) h, \quad h=\frac{T}{N+1}, \quad \text { for } n=-1, \ldots, N-1 .
$$

Using these discretization points, we employ the notation $u_{n}(\cdot) \approx u\left(\cdot, t_{n}\right)$ with the convention that $u_{-1}=0$. Applying standard approximations of the time-derivative in the heat equation, we obtain the following sequence of elliptic boundary value problems

$$
\Delta u_{n}-\gamma^{2} u_{n}=\sum_{m=0}^{n-1} \beta_{n-m} u_{m} \quad \text { in } D, \quad \text { for } n=0, \ldots, N-1,
$$

where $\gamma$ is a parameter that depends on the order of the approximation of the derivative. For the first order approximation of the time-derivative

$$
\gamma=\sqrt{\frac{1}{c h}}, \quad \beta_{i}=\left\{\begin{array}{cl}
-\frac{1}{c h}, & i=1 \\
0, & i>1
\end{array}\right.
$$

For the second order approximation

$$
\gamma=\sqrt{\frac{2}{c h}}, \quad \beta_{i}=(-1)^{i} \frac{4}{c h}
$$

Clearly there is a possibility to use more advanced time approximations but this is deferred to a future study, since in this paper we prefer a rather simple and fast approach to speed up the iterations of the iterative procedure. 
To reduce the elliptic problems (3.2) to boundary integral equations, we represent the solutions in the form of modified single- and double-layer potentials,

$$
\begin{aligned}
u_{n}(x) & =\frac{1}{\pi} \sum_{m=0}^{n} \int_{\Gamma_{1}} \varphi_{m}^{1}(y) \Phi_{n-m}(x, y) d s(y) \\
& +\frac{1}{\pi} \sum_{m=0}^{n} \int_{\Gamma_{2}} \varphi_{m}^{2}(y) \frac{\partial \Phi_{n-m}(x, y)}{\partial \nu(y)} d s(y), x \in D .
\end{aligned}
$$

Here $\varphi_{m}^{\ell}, m=0, \ldots, N-1, \ell=1,2$, are unknown densities and $\Phi_{m}, m=$ $0, \ldots, N-1$, are fundamental solutions of the sequence of elliptic equations in (3.2). These functions have the form $[4,6]$

$$
\Phi_{n}(x, y)=K_{0}(\gamma|x-y|) v_{n}(|x-y|)+K_{1}(\gamma|x-y|) w_{n}(|x-y|),
$$

where $K_{0}$ and $K_{1}$ are modified Bessel functions and we used the following polynomials

$$
v_{n}(r)=\sum_{m=0}^{\left[\frac{n}{2}\right]} a_{n, 2 m} r^{2 m}, \quad w_{n}(r)=\sum_{m=0}^{\left[\frac{n-1}{2}\right]} a_{n, 2 m+1} r^{2 m+1}
$$

for $n=0,1, \ldots, N-1\left(w_{0}=0\right)$ with $a_{n, 0}=1$ and the remaining coefficients recursively defined through

$$
\begin{gathered}
a_{n, n}=-\frac{1}{2 \gamma n} \beta_{1} a_{n-1, n-1}, \\
a_{n, k}=\frac{1}{2 \gamma k}\left\{4\left[\frac{k+1}{2}\right]^{2} a_{n, k+1}-\sum_{m=k-1}^{n-1} \beta_{n-m} a_{m, k-1}\right\}, \quad k=n-1, \ldots, 1 .
\end{gathered}
$$

To find the densities $\varphi_{m}^{1}$ and $\varphi_{m}^{2}$, we match the boundary conditions using theorems about continuous extension of single- and double-layer potentials to the boundary, and obtain the following system of integral equations

$$
\left\{\begin{array}{l}
\frac{1}{\pi} \int_{\Gamma_{1}} \varphi_{n}^{1}(y) \Phi_{0}(x, y) d s(y)+\frac{1}{\pi} \int_{\Gamma_{2}} \varphi_{n}^{2}(y) \frac{\partial \Phi_{0}(x, y)}{\partial \nu(y)} d s(y)=G_{n}^{1}(x), x \in \Gamma_{1}, \\
\frac{1}{\pi} \int_{\Gamma_{1}} \varphi_{n}^{1}(y) \frac{\partial \Phi_{0}(x, y)}{\partial \nu(x)} d s(y)+\frac{1}{\pi} \frac{\partial}{\partial \nu(x)} \int_{\Gamma_{2}} \varphi_{n}^{2}(y) \frac{\partial \Phi_{0}(x, y)}{\partial \nu(y)} d s(y)=G_{n}^{2}(x), x \in \Gamma_{2},
\end{array}\right.
$$


for $n=0, \ldots, N-1$, with right-hand sides

$$
\begin{aligned}
G_{n}^{1}(x) & =f_{n}^{1}(x)-\frac{1}{\pi} \sum_{m=0}^{n-1} \int_{\Gamma_{1}} \varphi_{m}^{1}(y) \Phi_{n-m}(x, y) d s(y) \\
& -\frac{1}{\pi} \sum_{m=0}^{n-1} \int_{\Gamma_{2}} \varphi_{m}^{2}(y) \frac{\partial \Phi_{n-m}(x, y)}{\partial \nu(y)} d s(y)
\end{aligned}
$$

and

$$
\begin{aligned}
G_{n}^{2}(x) & =f_{n}^{2}(x)-\frac{1}{\pi} \sum_{m=0}^{n-1} \int_{\Gamma_{1}} \varphi_{m}^{1}(y) \frac{\partial \Phi_{n-m}(x, y)}{\partial \nu(x)} d s(y) \\
& -\frac{1}{\pi} \sum_{m=0}^{n-1} \frac{\partial}{\partial \nu(x)} \int_{\Gamma_{2}} \varphi_{m}^{2}(y) \frac{\partial \Phi_{n-m}(x, y)}{\partial \nu(y)} d s(y) .
\end{aligned}
$$

The obtained system of boundary integral equations of the first kind is well-posed in various spaces; the following result about solvability of (3.6) holds in Hölder spaces [6].

Theorem 3.1 For any sequences $f_{n}^{1}$ in $C^{1, \alpha}\left(\Gamma_{1}\right)$ and $f_{n}^{2}$ in $C^{0, \alpha}\left(\Gamma_{2}\right)$, the system (3.6) possesses a unique solution $\varphi_{n}^{1}$ in $C^{0, \alpha}\left(\Gamma_{1}\right)$ and $\varphi_{n}^{2}$ in $C^{1, \alpha}\left(\Gamma_{2}\right)$.

Note that the first integral equation in (3.6) contains kernels with a logarithmic singularity and the corresponding integrals exist as improper integrals. The second integral equation in (3.6) contains a hypersingularity and this integral is interpreted as a Hadamard finite part integral (an equivalent interpretation in this case is to take the limiting value on the boundary of the normal derivative of the double-layer potential).

We assume that $\Gamma_{1}$ and $\Gamma_{2}$ can be represented by a parameterization of the form

$$
\Gamma_{\ell}=\left\{x_{\ell}(t)=\left(x_{1}^{\ell}(t), x_{2}^{\ell}(t)\right), 0 \leq t \leq 2 \pi\right\}, \ell=1,2 .
$$

Taking into account the parameterization of the two boundary parts we rewrite the system (3.6) in the parametric form as follows

$$
\left\{\begin{array}{l}
\frac{1}{2 \pi} \int_{0}^{2 \pi} \mu_{n}^{1}(\sigma) H_{0}^{11}(s, \sigma) d \sigma+\frac{1}{2 \pi} \int_{0}^{2 \pi} \mu_{n}^{2}(\sigma) H_{0}^{12}(s, \sigma) d \sigma=G_{n}^{1}(s), \\
\frac{1}{2 \pi} \int_{0}^{2 \pi} \mu_{n}^{1}(\sigma) H_{0}^{21}(s, \sigma) d \sigma+\frac{1}{2 \pi} \int_{0}^{2 \pi}\left\{\begin{array}{c}
\dot{\mu}_{n}^{2}(\sigma) \cot \frac{\sigma-s}{2}+ \\
\mu_{n}^{2}(\sigma) H_{0}^{22}(s, \sigma)
\end{array}\right\} d \sigma=G_{n}^{2}(s),
\end{array}\right.
$$


where $s \in[0,2 \pi], \mu_{n}^{1}(s)=\varphi_{n}^{1}\left(x_{1}(s)\right), \mu_{n}^{2}(s)=\sum_{m=0}^{n} \varphi_{n}^{2}\left(x_{2}(s)\right), n=0, \ldots, N-1$, and

$$
G_{n}^{1}(s)=f_{n}^{1}(s)-\frac{1}{2 \pi} \sum_{m=0}^{n-1} \int_{0}^{2 \pi}\left[\mu_{m}^{1}(\sigma) H_{n-m}^{11}(s, \sigma)+\psi_{m}^{2}(\sigma) H_{n-m}^{12}(s, \sigma)\right] d \sigma
$$

and

$$
G_{n}^{2}(s)=f_{n}^{2}(s)-\frac{1}{2 \pi} \sum_{m=0}^{n-1} \int_{0}^{2 \pi}\left[\mu_{m}^{1}(\sigma) H_{n-m}^{21}(s, \sigma)+\psi_{m}^{2}(\sigma) H_{n-m}^{22}(s, \sigma)\right] d \sigma
$$

with $f_{n}^{1}(s)=f_{n}^{1}\left(x_{1}(s)\right), f_{n}^{2}(s)=f_{n}^{2}\left(x_{2}(s)\right)\left|x_{2}^{\prime}(s)\right|, \psi_{n}^{2}(s)=\varphi_{n}^{2}\left(x_{2}(s)\right)$.

Here, we have used the Maue type expansion [12, p.117] for our analogue of the double layer potentials

$$
\begin{gathered}
\sum_{m=0}^{n} \frac{\partial}{\partial \nu(x)} \int_{\Gamma_{2}} \varphi_{m}^{2}(y) \frac{\partial \Phi_{n-m}(x, y)}{\partial \nu(y)} d s(y)=\sum_{m=0}^{n} \frac{\partial}{\partial \theta(x)} \int_{\Gamma_{2}} \frac{\partial \varphi_{m}^{2}}{\partial \theta}(y) \Phi_{n-m}(x, y) d s(y) \\
-\sum_{m=0}^{n} \int_{\Gamma_{2}} \varphi_{m}^{2}(y) \sum_{k=0}^{n-m} \beta_{n-m-k} \Phi_{k}(x, y)\langle\nu(x), \nu(y)\rangle d s(y), \quad x \in \Gamma_{2},
\end{gathered}
$$

where $\theta$ is the unit tangent vector to $\Gamma_{2}$ and by $\langle\cdot, \cdot\rangle$ we denote the scalar product in $\mathbb{R}^{2}$. Then we extract the hypersingularity in the form of a cot- weight function (for details we refer to [6]) and extracting the logarithmic singularities give the following representation

$$
H_{n}^{\ell \ell}(s, \sigma)=H_{n 0}^{\ell \ell}(s, \sigma) \ln \frac{4}{e} \sin ^{2} \frac{s-\sigma}{2}+H_{n 1}^{\ell \ell}(s, \sigma), \quad \ell=1,2, n=0, \ldots, N-1,
$$

where the functions $H_{n 0}^{\ell \ell}$ and $H_{n 1}^{\ell \ell}$ are continuous and their smoothness depends on the smoothness of the boundaries $\Gamma_{\ell}, \ell=1,2$.

\subsection{Full discretization}

For the numerical solution of the integral equations (3.8) we use a quadrature method based on trigonometric interpolation with equidistant grid points [5]. For this method, we choose $M \in \mathbb{N}$ and an equidistant mesh by setting

$$
s_{k}=\frac{k \pi}{M}, \quad k=0, \ldots, 2 M-1,
$$

and use the following quadrature rules

$$
\frac{1}{2 \pi} \int_{0}^{2 \pi} g(\sigma) \ln \left(\frac{4}{e} \sin ^{2} \frac{s_{j}-\sigma}{2}\right) d \sigma \approx \sum_{k=0}^{2 M-1} R_{|j-k|} g\left(s_{k}\right),
$$




$$
\begin{gathered}
\frac{1}{2 \pi} \int_{0}^{2 \pi} g^{\prime}(\sigma) \cot \frac{\sigma-s_{j}}{2} d \sigma \approx \sum_{k=0}^{2 n-1} T_{|j-k|} g\left(s_{k}\right), \\
\frac{1}{2 \pi} \int_{0}^{2 \pi} g(\sigma) d \sigma \approx \frac{1}{2 M} \sum_{k=0}^{2 M-1} g\left(s_{k}\right) .
\end{gathered}
$$

with the known weights $R_{j}$ and $T_{j}[5,6,12]$. Note here that the first two quadratures are for the integrals with logarithmic- and hypersingularities. They are obtained by the trigonometrical interpolation of the smooth part of integrand $g$ and together with exact (analytical) integration.

We then use the quadrature rules (3.9)-(3.11) to approximate the three types of integrals in (3.8) and collocate the approximated equations at the nodal points to obtain the following recurrence sequence of linear systems

$$
\left\{\begin{array}{l}
\sum_{k=0}^{2 M-1}\left[\mu_{n, k}^{1}\left\{\begin{array}{c}
R_{|j-k|} H_{00}^{11}\left(s_{j}, s_{k}\right) \\
+\frac{1}{2 M} H_{01}^{11}\left(s_{j}, s_{k}\right)
\end{array}\right\}+\mu_{n, k}^{2} \frac{1}{2 M} H_{0}^{12}\left(s_{j}, s_{k}\right)\right]=G_{n, j}^{1}, \\
\sum_{k=0}^{2 M-1}\left[\mu_{n, k}^{1} \frac{1}{2 M} H_{0}^{21}\left(s_{j}, s_{k}\right)+\mu_{n, k}^{2}\left\{\begin{array}{c}
T_{|j-k|}+R_{|j-k|} H_{00}^{22}\left(s_{j}, s_{k}\right) \\
+\frac{1}{2 M} H_{01}^{22}\left(s_{j}, s_{k}\right)
\end{array}\right\}\right]=G_{n, j}^{2},
\end{array}\right.
$$

for $j=0, \ldots, 2 M-1, n=0, \ldots, N-1$, which we have to solve for the nodal values $\mu_{n, j}^{\ell}$ of the approximating trigonometric polynomial $\mu_{n, M}^{\ell}$ in the space of trigonometrical polynomials $\mathcal{T}_{M}$ of degree $M$. Of course, the approximate values $G_{n, j}^{\ell}$ for the right-hand side are also obtained using (3.9) and (3.11). The following result about the convergence and error estimate for this method was recently proved in $[6]$

Theorem 3.2 For $\Gamma_{1}, \Gamma_{2} \in C^{\ell+2}, \ell \geq 1, f_{n}^{1} \in C^{\ell, \beta}[0,2 \pi], f_{n}^{2} \in C^{\ell-1, \beta}[0,2 \pi]$ and for a sufficiently large $M$, the system (3.12) has a unique solution for every $n=0, \ldots, N-1$. For the exact solution $\tilde{\mu}_{n}=\left(\mu_{n}^{1}, \mu_{n}^{2}\right)$ to (3.8) and approximate solution $\tilde{\mu}_{n, M}=\left(\mu_{n, M}^{1}, \mu_{n, M}^{2}\right) \in \mathcal{T}_{M} \times \mathcal{T}_{M}$, we have the error estimates

$$
\left\|\tilde{\mu}_{n}-\tilde{\mu}_{n, M}\right\|_{m, \alpha} \leq C_{n} \frac{\ln M}{M^{\ell-m+\beta-\alpha}}\left\|\tilde{\mu}_{n}\right\|_{\ell, \beta}
$$

for $0 \leq m \leq \ell, 0<\alpha \leq \beta<1$ and some constants $C_{k}$ depending only on $\alpha, \beta, m, \ell$.

\subsection{Cauchy data calculation}

The iterative procedure given in Section 2 for the Cauchy problem (1.1) involves the calculation of the temperature or heat flux on the various boundary parts at each iteration step. In order to obtain formulas for the traces of the solution and its normal derivative on the boundaries, we apply theorems on jump relations for single- and double-layer potentials. 
On the internal boundary $\Gamma_{1}$ we have

$$
\begin{aligned}
\frac{\partial u_{n}}{\partial \nu}(x)=-\sum_{m=0}^{n} \varphi_{m}^{1}(x) & +\frac{1}{\pi} \sum_{m=0}^{n} \int_{\Gamma_{1}} \varphi_{m}^{1}(y) \frac{\partial}{\partial \nu(x)} \Phi_{n-m}(x, y) d s(y) \\
& +\frac{1}{\pi} \sum_{m=0}^{n} \int_{\Gamma_{2}} \varphi_{m}^{2}(y) \frac{\partial^{2}}{\partial \nu(x) \nu(y)} \Phi_{n-m}(x, y) d s(y), \quad x \in \Gamma_{1}
\end{aligned}
$$

and on the external boundary $\Gamma_{2}$

$$
\begin{aligned}
u_{n}(x) & =\frac{1}{\pi} \sum_{m=0}^{n} \int_{\Gamma_{1}} \varphi_{m}^{1}(y) \Phi_{n-m}(x, y) d s(y)-\sum_{m=0}^{n} \varphi_{m}^{2}(x) \\
& +\frac{1}{\pi} \sum_{m=0}^{n} \int_{\Gamma_{2}} \varphi_{m}^{2}(y) \frac{\partial \Phi_{n-m}(x, y)}{\partial \nu(y)} d s(y), \quad x \in \Gamma_{2} .
\end{aligned}
$$

Using the parameterization (3.7) of the boundary parts and extracting the logarithmic singularities give the following representation

$$
\begin{aligned}
\frac{\partial u_{n}}{\partial \nu}\left(x_{1}(s)\right)= & \frac{1}{2 \pi} \sum_{m=0}^{n} \int_{0}^{2 \pi} \mu_{m}^{1}(\sigma)\left[\tilde{H}_{n-m, 0}^{11}(s, \sigma) \ln \frac{4}{e} \sin ^{2} \frac{s-\sigma}{2}+\tilde{H}_{n-m, 1}^{11}(s, \sigma)\right] d \sigma \\
& -\sum_{m=0}^{n} \mu_{m}^{1}(s)+\frac{1}{2 \pi} \sum_{m=0}^{n} \int_{0}^{2 \pi} \psi_{m}^{2}(\sigma) \tilde{H}_{n-m}^{12}(s, \sigma) d \sigma
\end{aligned}
$$

and

$$
\begin{aligned}
u_{n}\left(x_{2}(s)\right) & =\frac{1}{2 \pi} \sum_{m=0}^{n} \int_{0}^{2 \pi} \mu_{m}^{1}(\sigma) \tilde{H}_{n-m}^{21}(s, \sigma) d \sigma-\sum_{m=0}^{n} \psi_{m}^{2}(s) \\
& +\frac{1}{2 \pi} \sum_{m=0}^{n} \int_{0}^{2 \pi} \psi_{m}^{2}(\sigma)\left[\tilde{H}_{n-m, 0}^{22}(s, \sigma) \ln \frac{4}{e} \sin ^{2} \frac{s-\sigma}{2}+\tilde{H}_{n-m, 1}^{22}(s, \sigma)\right] d \sigma
\end{aligned}
$$

for $s \in[0,2 \pi]$. Here, the kernels $\tilde{H}_{n, 0}^{\ell \ell}, \tilde{H}_{n, 1}^{\ell \ell}, \ell=1,2, \tilde{H}_{n}^{12}$ and $\tilde{H}_{n}^{21}$ are smooth. Thus, we can apply the quadrature rules (3.9) and (3.11).

\section{Numerical examples}

We present some numerical examples for the above described iterative procedure for finding the temperature $u$ to (1.1). To generate data, the Cauchy problem (1.1) are synthesized with the help of the following direct mixed initial boundary value 
problem

$$
\begin{cases}\frac{1}{c} \frac{\partial g}{\partial t}=\Delta g & \text { in } D \times(0, T), \\ g=g_{1} & \text { on } \Gamma_{1} \times(0, T), \\ \frac{\partial g}{\partial \nu}=g_{2} & \text { on } \Gamma_{2} \times(0, T), \\ g(x, 0)=0 & \text { for } x \in D,\end{cases}
$$

for some given input data $g_{1}$ and $g_{2}$ that will be explicitly specified in each of

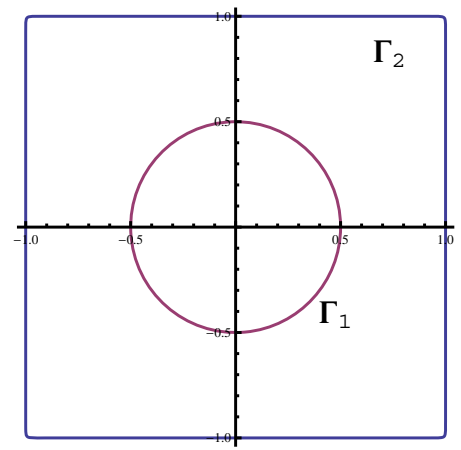

a). The solution domain $D$

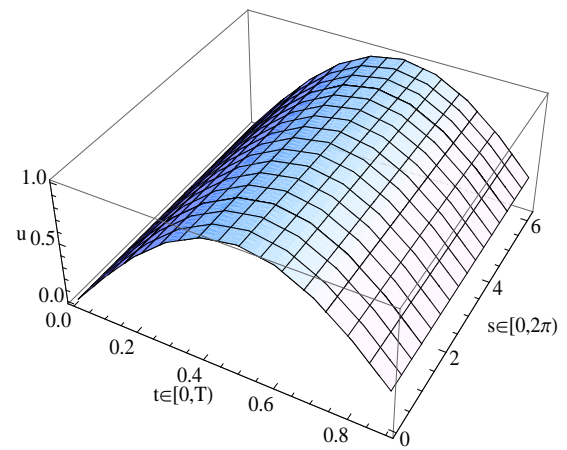

b). The exact value of the temperature $\left.u\right|_{\Gamma_{1} \times(0, T)}$.

Figure 2: Domain and exact solution for Ex. 1.

the two examples below. Once $g_{1}$ and $g_{2}$ have been specified we can calculate the value of the trace of the solution $g$ of this mixed problem on the external boundary $\Gamma_{2}$, i.e. we can select the Cauchy data in (4.1) as $f_{1}=\left.g\right|_{\Gamma_{2}}$ and $f_{2}=g_{2}$. We then apply the procedure to reconstruct the temperature on the inner boundary $\Gamma_{1}$ and compare it with the given element $g_{1}$. In order to not perform an "inverse crime", the direct problem (4.1) used to generate data is solved on a finer mesh and noise will be added to the data as well.

Ex. 1. For the solution domain, we select a doubly connected domain with the outer boundary being of rectangular shape with slightly rounded corners and having parameterization

$$
\Gamma_{2}=\left\{\left[(\cos s)^{100}+(\sin s)^{100}\right]^{-0.01}(\cos s, \sin s), s \in[0,2 \pi]\right\}
$$

and the inner boundary is a circle with parameterization (see Figure 2a)

$$
\Gamma_{1}=\{0.5(\cos s, \sin s), s \in[0,2 \pi]\} .
$$

We choose these boundary parts to be smooth to avoid any additional difficulties with possible singularities near corner points. As explained at the end of Section 2, in case of singularities the method [8] can be employed. To generate Cauchy data, we assume that the heat flux is zero on the outer boundary $\Gamma_{2}$, which means 


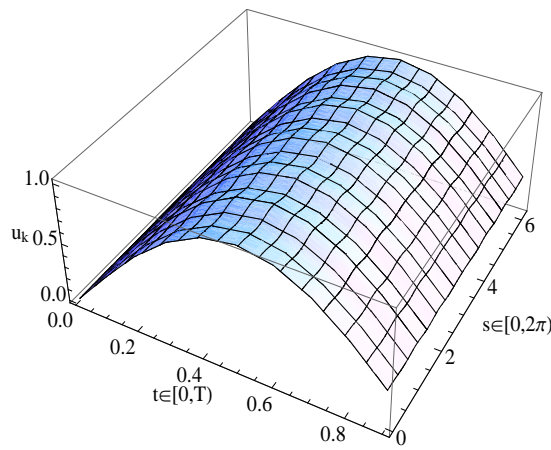

a). Exact data

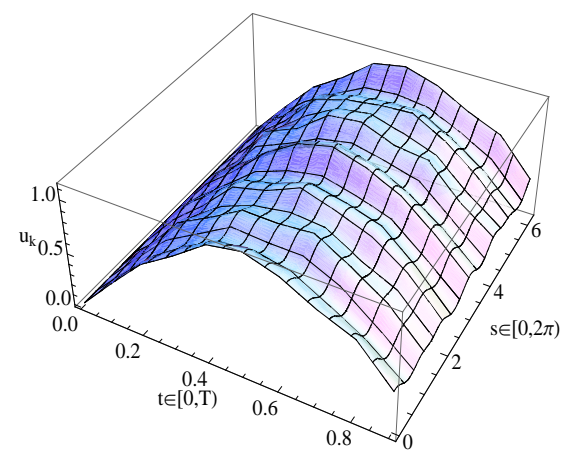

b). $5 \%$ noise

Figure 3: Numerical solution for Ex. 1.

that $f_{2}(x, t)=0$ on $\Gamma_{2}$. The temperature on $\Gamma_{2}, f_{1}(x, t)$, is generated from the above direct problem (4.1) with $g_{1}(x, t)=\sin (\pi t)$ and $g_{2}(x, t)=f_{2}(x, t)=0$. The final time $T$ is chosen to $T=1$, the thermal diffusivity is $c=1$, and $\gamma_{L}=$ 1.0. For the discretization in time (3.1) we take $N=9$ and for discretization in space $M=64$ in the quadrature method of Section 3.2. Employing the iterative

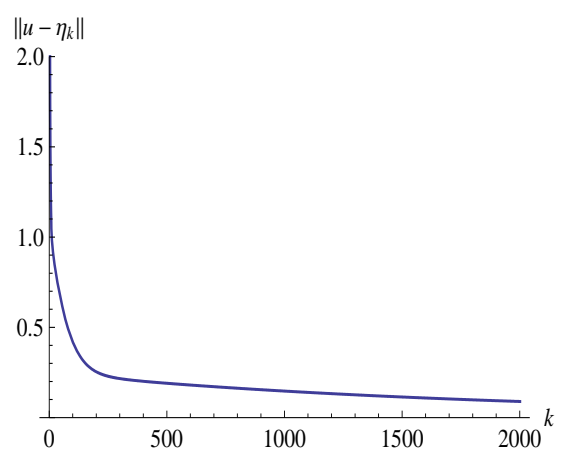

a). Exact data

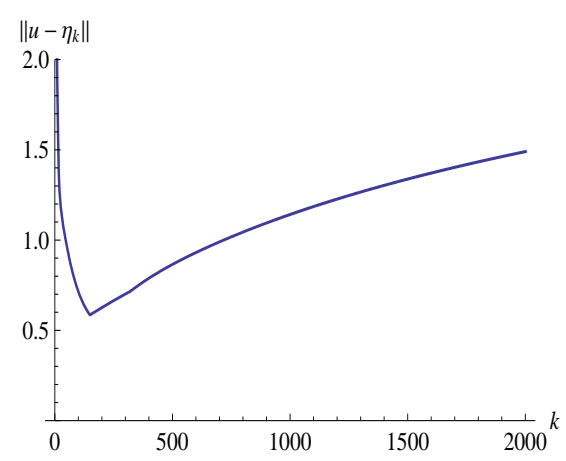

b). $5 \%$ noise

Figure 4: The errors $\left\|u-\eta_{k}\right\|_{L_{2}\left(\Gamma_{1} \times(0, T)\right)}$ for Ex. 1.

procedure with exact (numerical) data and initial element $\eta_{0}=0$, we obtain an accurate approximation after about 2000 iterations. The reconstruction on the inner boundary of the temperature after 2000 iterations, that is $\left.u_{k}\right|_{\Gamma_{1} \times(0, T)}=\eta_{k}$, $k=2000$, is shown in Figure 3a.

We then added $5 \%$ gaussian noise to the function $g_{1}$. Due to the ill-posedness of the Cauchy problem (1.1), this error will start to magnify after a certain number of iterations. To stop the iterations, the discrepancy principle [13] is employed generating the stopping index to be $k=190$. The corresponding temperature reconstruction is shown in Figure 3b. Adding more noise gives a less accurate ap- 
proximation as expected, however, it is still a stable and reasonable approximation. The corresponding error plots are also given, see Figure 4.

Ex. 2. In this example we change the solution domain and the boundary functions. Let the outer boundary $\Gamma_{2}$ be the unit circle and as the inner boundary $\Gamma_{1}$, we take a peanut shaped inclusion given as (see Figure $5 \mathrm{a}$ )

$$
\Gamma_{1}=\{\sqrt{\cos s+0.25 \sin s}(\cos s, \sin s), s \in[0,2 \pi]\} .
$$

To generate the temperature function $f_{1}(x, t)$ in the Cauchy data, we take $g_{1}(x, t)=$

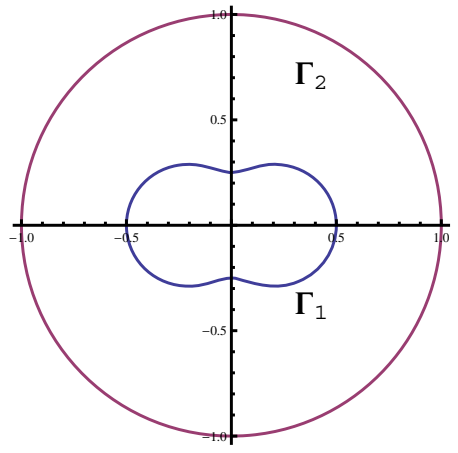

a). The solution domain $D$

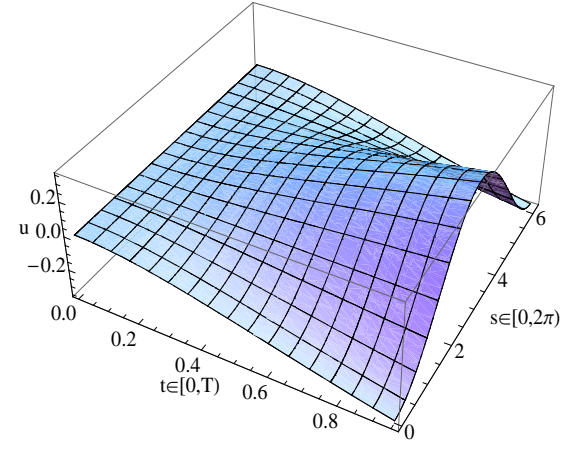

b). The exact value of the temperature $\left.u\right|_{\Gamma_{1} \times(0, T)}$.

Figure 5: Domain and exact solution for Ex. 2.

$-t^{2} \exp (-t) \cos (x)$ and $g_{2}(x, t)=f_{2}(x, t)=0$, and solve the above direct problem. The parameters remain the same as in the previous example.

Employing the iterative procedure with exact (numerical) data and initial element $\eta_{0}=0$, we obtain also in this example an accurate approximation after about 2000 iterations. The reconstruction on the inner boundary of the temperature after 2000 iterations, that is $\left.u_{k}\right|_{\Gamma_{1} \times(0, T)}=\eta_{k}, k=2000$, is shown in Figure 5 .

We then added $5 \%$ gaussian noise to the function $g_{1}$. Due to the ill-posedness of the Cauchy problem (1.1), this error will start to magnify after a certain number of iterations. To stop the iterations, the discrepancy principle [13] is employed generating the stopping index to be $k=651$, which is larger than in the previous example. This is probably due to the more complicated shape of the inner boundary $\Gamma_{1}$ making the procedure more time-consuming. The corresponding temperature reconstruction is shown in Figure 6. Adding more noise gives a less accurate approximation as expected, however, it is still a stable and reasonable approximation. The corresponding error plots are also given, see Figure 7.

\section{Conclusion}

After implementing the above iterative method for the Cauchy problem (1.1) for the two examples presented we came to the following conclusions: 


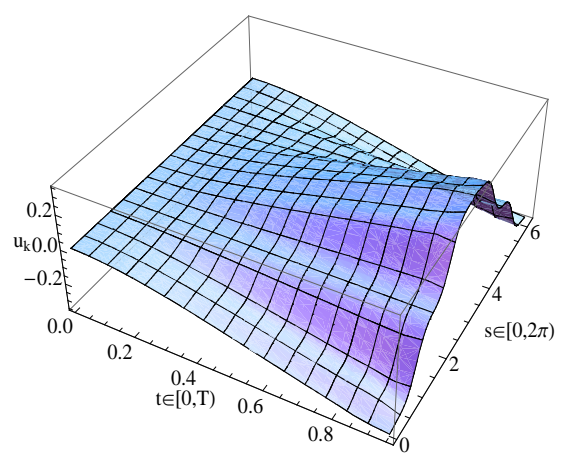

a). Exact data

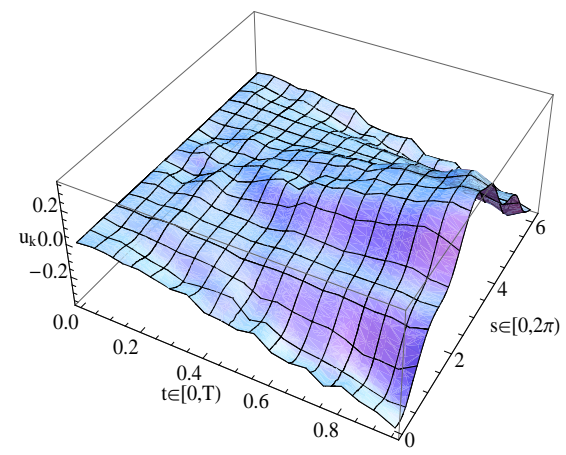

b). $5 \%$ noise

Figure 6: Numerical solution for Ex. 2.

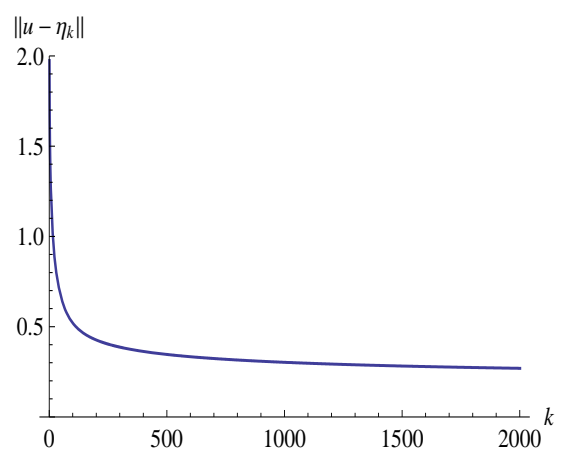

a). Exact data

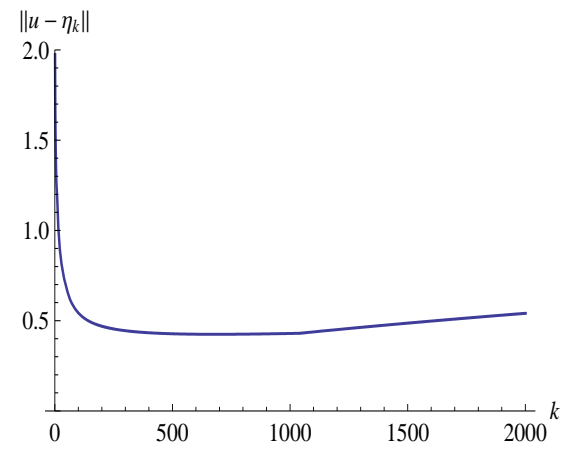

b). $5 \%$ noise

Figure 7: The errors $\left\|u-\eta_{k}\right\|_{L_{2}\left(\Gamma_{1} \times(0, T)\right)}$ for Ex. 2.

- The combination of the Landweber procedure, the method of Rothe and boundary integral equations give an accurate and acceptable solution to the time-dependent Cauchy problem (1.1) for the heat equation.

- In order to obtain a stable solution of direct initial boundary value problems for the heat equation used in the iterative procedure, the value of discretization parameter in the spatial variable should be consistent with the value of discretization parameter in time. This in particular implies that when increasing the amount of points in the time discretization (3.1) we should similarly choose more collocation points on the boundary parts.

\section{References}

[1] S. Andrieux, T. N. Baranger and A. Ben Abda Solving Cauchy problems by minimizing an energy-like functional, Inverse Problems 22, 135-133 (2006). 
[2] G. Bastay, V. A. Kozlov and B. O. Turesson Iterative methods for an inverse heat conduction problem, J. Inverse Ill-posed Probl. 9, 375-388 (2001).

[3] R. Chapko and B. T. Johansson An alternating boundary integral based method for a Cauchy problem for Laplace equation in semi-infinite domains, Inverse Probl. Imaging. 3, 317-333 (2008).

[4] R. Chapko and R. Kress Rothe's Method for the Heat Equation and Boundary Integral Equations, J. Integral Equations Appl. 9, 47-69 (1997).

[5] R. Chapko and R. Kress On a quadrature method for a logarithmic integral equation of the first kind. In Agarwal, ed.: World Scientific Series in Applicable Analysis.- Vol. 2. Contributions in Numerical Mathematics.-World Scientific, Singapore. 127-140 (1993).

[6] R. S. Chapko and V. G. Vavrychuk On the numerical solution of a mixed initial boundary value problem for the heat equation in a double-connected planar domain, J. Numer. Appl. Math. 97, 26-38 (2009).

[7] Dinh Nho Hào Methods for Inverse Heat Conduction Problems, Peter Lang Verlag Frankfurt/Main, Bern, New York, Paris (1998).

[8] B. T. Johansson An iterative method for a Cauchy problem for the heat equation, IMA J. Appl. Math 71, 262-286 (2006).

[9] V. A. Kondrat'ev The smoothness of a solution of Dirichlet's problem for second-order elliptic equations in a region with a piecewise-smooth boundary, Differ. Uravn. 6, 1831-1843 (1970). English translation: Differ. Equ. 6, 13921401 (1970).

[10] V. A. Kozlov and V. G. Maz'ya On iterative procedures for solving ill-posed boundary value problems that preserve differential equations, Algebra i Analiz 1, 144-170 (1989). English translation: Leningrad Mathematics Journal 1, 12071228 (1991).

[11] V. A. Kozlov, V. G. Mazya and V.A. Fomin An Iterative Method for Solving the Cauchy Problem for Elliptic Equations, Comput. Math. Phys. 1, 45-52 (1991).

[12] R. Kress Linear Integral Equations, 2nd. ed. Springer-Verlag, Heidelberg (1999).

[13] V. A. Morozov On the solution of functional equations by the method of regularization Doklady Mathematics 7, 414-417 (1966).

[14] H.-J. Reinhardt, Dinh Nho Hào, J. Frohne and F.-T. Suttmeier Numerical solution of inverse heat conduction problems in two spatial dimensions, J. Inverse and Ill-Posed Problems 15, 181-198 (2007).

[15] J.-C. Saut and B. Scheurer Unique continuation for some evolution equations, J. Differential Equations 66, 118-139 (1987). 